\title{
Papers
}

\section{Prospective cohort study of cannabis use, predisposition for psychosis, and psychotic symptoms in young people}

Cécile Henquet, Lydia Krabbendam, Janneke Spauwen, Charles Kaplan, Roselind Lieb, Hans-Ulrich Wittchen, Jim van Os

\begin{abstract}
Objective To investigate the relation between cannabis use and psychotic symptoms in individuals with above average predisposition for psychosis who first used cannabis during adolescence.

Design Analysis of prospective data from a population based sample. Assessment of substance use, predisposition for psychosis, and psychotic symptoms was based on standardised personal interviews at baseline and at follow up four years later. Participants 2437 young people (aged 14 to 24 years) with and without predisposition for psychosis.

Main outcome measure Psychotic symptoms at follow up as a function of cannabis use and predisposition for psychosis at baseline.

Results After adjustment for age, sex, socioeconomic status, urbanicity, childhood trauma, predisposition for psychosis at baseline, and use of other drugs, tobacco, and alcohol, cannabis use at baseline increased the cumulative incidence of psychotic symptoms at follow up four years later (adjusted odds ratio $1.67,95 \%$ confidence interval 1.13 to 2.46 ). The effect of cannabis use was much stronger in those with any predisposition for psychosis at baseline $(23.8 \%$ adjusted difference in risk, $95 \%$ confidence interval 7.9 to 39.7 , $\mathrm{P}=0.003)$ than in those without $(5.6 \%, 0.4$ to $10.8, \mathrm{P}=0.033)$.

The risk difference in the "predisposition" group was significantly greater than the risk difference in the "no predisposition" group (test for interaction 18.2\%, 1.6 to 34.8 , $\mathrm{P}=0.032$ ). There was a dose-response relation with increasing frequency of cannabis use. Predisposition for psychosis at baseline did not significantly predict cannabis use four years later (adjusted odds ratio 1.42, 95\% confidence interval 0.88 to 2.31).

Conclusion Cannabis use moderately increases the risk of psychotic symptoms in young people but has a much stronger effect in those with evidence of predisposition for psychosis.
\end{abstract}

\section{Introduction}

There is accumulating and converging evidence that cannabis use may be a risk factor for psychotic symptoms. ${ }^{12}$ The possible causal nature of the association between cannabis and psychosis, however, is still a matter of debate, the main discussion revolving around the role of predisposition for psychosis and adjustment for confounders. ${ }^{3}$ According to the self medication hypothesis, ${ }^{5}$ individuals may start using cannabis because of predisposition for psychosis, rather than cannabis use causing expression of psychosis. To our knowledge, no prospective study to date has tested this hypothesis using information on predisposition for psychosis in relation to later cannabis use. Recent research, however, has suggested that rather than being the cause of cannabis use, such predisposition interacts synergistically with cannabis use. Two studies suggested that the joint effect of cannabis use and predisposition for psychosis on the emergence of psychotic symptoms was greater than the sum of their individual effects. ${ }^{26}$

We investigated prospectively whether cannabis use at baseline increases the risk of subsequent development of psychotic symptoms, whether any such increase in risk is higher in individuals with a predisposition for psychosis, and whether baseline expression predisposition increases the risk for subsequent use of cannabis. We adjusted for possible confounding factors in a large population-based sample of adolescents and young adults.

\section{Methods}

Sample

The study was part of the early developmental stages of psychopathology (EDSP) study, ${ }^{7}$ in which data were collected on the prevalence, incidence, risk factors, comorbidity, and four year course of mental disorders in a random regional representative population sample of adolescents and young adults aged 14-24 years. The EDSP study is prospective, consisting of a baseline survey in 1995, an assessment of a subsample in 1996-7, and a four year follow up of the total sample in 1999.' The current analyses used the baseline and four year follow up data. The study sample was randomly drawn from the respective population registry offices of the city and each of the 29 counties of Munich. The base population were all those born between 1 June 1970 and 31 May 1981 who were registered as living in these areas as their primary place of residence and were German citizens. At baseline 3021 participants were interviewed face to face in their homes by using a computer assisted method (response rate $71 \%$ ). An average of 42 months after the baseline investigations 2548 (response rate 84\%) participants were successfully followed up. Of these, 90 participants did not want to respond to questions about illicit drug use at baseline or follow up, and data on the psychosis section of the Munich version of the international composite interview were incomplete for 21 participants. Our analyses were therefore of 2437 participants. To have $80 \%$ power to detect an odds ratio of 2.5 at the $5 \%$ level, given a base rate of psychotic experiences of $10 \%$ and a ratio of unexposed to exposed of $6: 1,{ }^{2}{ }^{8}$ we needed a sample size of 616 . As around four times as many subjects are required for an inter- 
action such as between cannabis use and predisposition for psychosis, ${ }^{9}$ the sample size of 2437 was large enough.

\section{Instruments}

Participants were interviewed by trained psychologists using the Munich version of the composite international diagnostic interview (M-CIDI) ${ }^{10}$ At baseline, they used the lifetime version of the interview. The interval version was used at follow up, which refers to the period of assessment between baseline and follow up. We assessed data on the psychosis $(\mathrm{G})$ section only at follow up and these represent lifetime ratings. At baseline and at follow up, participants additionally completed the self report symptom checklist (SCL-90-R) $)^{11}$ to screen for a broad range of psychopathological experiences including psychosis.

We used the "paranoid ideation" and "psychoticism" subscales of the symptom checklist to explore predisposition for psychosis at baseline and at follow up. Total scores of both subscales were added into a total score. Participants with total scores above the 90th centile were considered as having a predisposition for psychosis, both at baseline and at follow up.

We defined an outcome of psychosis as at least one (broad psychosis outcome) or at least two (narrow psychosis outcome) positive ratings on any of the 15 core psychosis items of the M-CIDI. We used the narrow outcome to exclude the possibility of spurious results due to false positive misclassification. None of these psychotic symptoms was due to acute effects of medication, drugs, or alcohol.

To assess symptoms of depression at baseline and follow up we calculated a mean score of the ratings on the 28 items of the depression section (E) of the M-CIDI. We divided this into two groups at the 90th centile, yielding a measure of significant symptoms at both baseline and follow up.

We assessed use of cannabis and other substances using the $\mathrm{L}$-section of the M-CIDI. Individuals with lifetime cannabis use of five times or more at baseline were considered as exposed to cannabis. Cannabis exposure at baseline was defined as lifetime cannabis use of five times or more (any use) and frequency of use (use during the period of heaviest use: no use; less than once a month; three to four times a month; once to twice a week; three to four times a week; almost daily). Cannabis use at follow up was analysed as cannabis use of five times or more during the four years to follow up (any use at follow up). We combined psychostimulants, sedatives, opiates, cocaine, phencyclidine (PCP), and psychedelic drugs into a group of "other drugs." Lifetime tobacco use at baseline was defined as daily use during at least one month. Alcohol use at baseline was analysed as frequency of use during the past 12 months (no use; less than once a month; three to four times a month; once to twice a week; three to four times a week; almost daily).

\section{Statistical analyses}

Associations between any use of cannabis or frequency of use at baseline and psychotic symptoms at follow up were expressed as odds ratios from logistic regression models in Stata, release 8.0 (StataCorp, College Station, TX). All analyses were a priori adjusted for age, sex, socioeconomic status, urbanicity, ${ }^{12}$ and experience of childhood trauma ${ }^{13}$ as well as for predisposition for psychosis at baseline. To test whether the effect of cannabis on psychosis was independent of other drugs, tobacco, alcohol, and symptoms of depression at baseline and at follow up, we entered these variables in the model with any cannabis use at baseline. We also adjusted the effect of baseline cannabis on psychotic symptoms according to the M-CIDI at follow up for predisposition for psychosis at follow up.
The population attributable fraction was derived from the associations between any use of cannabis and psychotic symptoms according to the M-CIDI at follow up (adjusted for demographics and trauma during childhood) in the logistic regression models, both for the whole dataset and for the individuals with expression of baseline predisposition, using the aflogit procedure in Stata. This parameter gives a measure of the proportion of cases in participants with psychotic symptoms according to the M-CIDI at follow up that could have been prevented, assuming causality, had the exposure to cannabis been eliminated completely from the baseline population.

We calculated the interaction between predisposition for psychosis at baseline and any cannabis use (adjusted for demographics, childhood trauma, and predisposition at follow up) under an additive model rather than a multiplicative model because an additive model is more likely to yield information on the degree of synergism between causes-that is, the extent to which both causes depend on each other or coparticipate in disease causation. ${ }^{14}{ }^{15}$

We investigated the self medication hypothesis by calculating the association between predisposition for psychosis at baseline and cannabis use at follow up, both for the whole dataset and for the individuals who had not used cannabis at baseline. We used sensitivity analyses to examine whether differential attrition in the sample as a whole (3021 at baseline, 2437 at follow up) could have biased the findings. This was done by multiple imputation of missing values of cannabis use at baseline, predisposition for psychosis at baseline, and psychotic symptoms according to the M-CIDI at follow up with the hotdeck command in Stata.

\section{Results}

We followed up 2437 participants, of which 1251 (51.3\%) were men. The mean age was 18.3 years (SD 3.3 years) at baseline and 21.8 years (3.4 years) at follow up. At four year follow up the cumulative lifetime incidence of at least one psychotic symptom was $424(17.4 \%)$, irrespective of severity and impairment probe criteria, and $174(7.1 \%)$ participants reported two or more psychotic symptoms. At baseline $320(13.1 \%)$ admitted to any use of cannabis (five times or more) and $361(14.8 \%)$ did so at follow up.

Any cannabis use at baseline increased the risk of psychotic symptoms according to the M-CIDI at follow up four years later in a dose-response fashion (tables 1-3), regardless of confounders, and with larger effect sizes for the narrowly defined psychosis outcome.

The effect of baseline cannabis use on the psychosis outcome according to the M-CIDI at follow up four years later was much stronger in those with predisposition for psychosis at baseline (23.8\% adjusted difference in risk) than in those without $(5.6 \%$ adjusted difference in risk, table 4). The population attributable fraction was $6.2 \%$ for the total group and more than twice as large (14.2\%) for the group with predisposition for psychosis at baseline.

Predisposition for psychosis at baseline did not significantly predict cannabis use at follow up four years later (odds ratio 1.42, $95 \%$ confidence interval 0.94 to 2.15 , for the whole sample and $1.42,0.88$ to 2.31 , for the subgroup with no cannabis use at baseline).

Based on 1000 imputation sequences in which we stochastically imputed missing values of cannabis use at baseline and psychotic symptoms according to the M-CIDI at four year follow up in the whole sample, the estimated average additive interaction between predisposition for psychosis at baseline and cannabis 


\begin{tabular}{|c|c|c|c|c|}
\hline \multirow[b]{2}{*}{ Cannabis use at baseline } & \multicolumn{2}{|c|}{ Any psychotic symptom at follow up } & \multicolumn{2}{|c|}{ At least two psychotic symptoms at follow up } \\
\hline & Yes $(n=424)$ & No $(n=2013)$ & Yes $(n=174)$ & No $(n=2263)$ \\
\hline Any use ( $\geq 5$ times) & $82(19.3)$ & $238(11.8)$ & $44(25.3)$ & $276(12.2)$ \\
\hline \multicolumn{5}{|l|}{ Cumulative frequency*: } \\
\hline None & $342(80.7)$ & $1775(88.2)$ & $130(74.7)$ & $1987(87.8)$ \\
\hline$<1$ times/month & $13(3.1)$ & $69(3.4)$ & $5(2.9)$ & $77(3.4)$ \\
\hline 3-4 times/month & $18(4.2)$ & $62(3.1)$ & $10(5.7)$ & $70(3.1)$ \\
\hline 1-2 times/week & $17(4.0)$ & $40(2.0)$ & $7(4.0)$ & $50(2.2)$ \\
\hline 3-4 times/week & $12(2.8)$ & $21(1.0)$ & $8(4.6)$ & $25(1.1)$ \\
\hline Almost daily & $22(5.2)$ & $46(2.3)$ & $14(8.0)$ & $54(2.4)$ \\
\hline
\end{tabular}

Some percentages do not total 100 because of rounding.

use at baseline remained significant $(19.5 \%$ difference in risk, $95 \%$ confidence interval 0.3 to $38.6, \mathrm{P}=0.039$ ).

\section{Discussion}

Exposure to cannabis during adolescence and young adulthood increases the risk of psychotic symptoms later in life. The findings confirm earlier suggestions that this association is stronger for individuals with predisposition for psychosis $^{26}$ and stronger for the more severe psychotic outcomes. ${ }^{26}$ Frequent use of cannabis was associated with higher levels of risk in a dose-response fashion. Associations were independent of other variables known to increase the risk for psychosis. Also, the effect of cannabis remained significant after we corrected for baseline use of other drugs, tobacco, and alcohol. Finally, the data did not support the self medication hypothesis as baseline predisposition for psychosis did not significantly predict cannabis use at follow up.

\section{Strengths and weaknesses}

We examined psychotic symptoms according to the M-CIDI at follow up in a non-clinical sample. Symptoms were more prevalent than psychotic disorders defined according to the Diagnostic and Statistic Manual of Mental Disorders, fourth edition, but nevertheless have been shown to be on the same continuum of experiences as more severe states of psychosis, such as schizophrenia. ${ }^{816}$ Established risk factors for schizophrenia, such as urbanicity and familial predisposition, also affect the occurrence of psychotic symptoms. ${ }^{15}{ }^{17}$ Our results confirm those from three previous studies that showed that exposure to cannabis plays a part not only in the expression of psychotic disorder but also in the emergence of less severe psychotic experiences. ${ }^{2}{ }^{6} 18$

At baseline we used self reported psychotic experiences on the symptom checklist to determine predisposition for psychosis, whereas at follow up four years later we used the M-CIDI to determine psychosis outcome. In the group with predisposition for psychosis at baseline, any effect of cannabis can thus be interpreted as psychosis persisting from baseline to follow up (if we assume that the two measures of psychosis are identical), rather than an effect of transition from expression of predisposition at baseline to expression of overt symptoms at follow up. Although both explanations would be equally impor- tant, adjustment for the effect of the follow up equivalent of the baseline measure of predisposition for psychosis did not change the observed association between cannabis and psychotic symptoms according to the M-CIDI, indicating that the effect of cannabis can be interpreted as onset of clinical psychosis outside the continuity between the measure of predisposition for psychosis at baseline and follow up. In addition, cannabis also had a significant effect on psychotic symptoms in the group without predisposition for psychosis at baseline, albeit of smaller effect.

\section{Synergistic interaction between cannabis and predisposition} for psychosis

Findings from earlier research suggest biological plausibility, involving the endocannabinoid CB1 receptor system in close interaction with the dopamine neurotransmitter system. For example, delta-9-tetrahydrocannabinol (the major psychoactive component of cannabis) increases presynaptic dopamine efflux and utilisation in the prefrontal cortex in rats. ${ }^{19}$ Increased concentrations of $\mathrm{CB} 1$ receptors were found in the dorsolateral prefrontal cortex of patients with schizophrenia, ${ }^{20}$ and concentrations of anandamide (an endogenous cannabinoid that binds to the $\mathrm{CB} 1$ receptor) were found to be higher in the cerebrospinal fluid of these patients. ${ }^{21}$ Furthermore, there is evidence from animal studies that puberty in rats is a vulnerable period with respect to the adverse effects of cannabinoids. ${ }^{22}$ The longlasting behavioural disturbances observed in this animal study are consistent with impairments generally found in patients with schizophrenia, and the changes in these rats were reversed by the acute administration of the dopamine antagonist haloperidol. ${ }^{22}$ Repeated exposure to cannabis may cause initial increases in synaptic dopamine and then lead to more prolonged changes in the endogenous cannabinoid systems. These changes might be most profound after exposure to cannabis during adolescence and in individuals with a pre-existing vulnerability to dysregulation of the cannabinoid system and related neurotransmission systems.

This work is part of the early developmental stages of psychopathology (EDSP) study. Principal investigators are Hans-Ulrich Wittchen and Roselind Lieb. Current or former staff members of the EDSP group include Kirsten von Sydow, Gabriele Lachner, Axel Perkonigg, Peter Schuster, Michael Höfler, Holger Sonntag, Esther Beloch, Martina Fuetsch, Elzbieta Garczynski, Alexandra Holly, Barbara Isensee, Chris Nelson, Hildegard

Table 2 Associations between any cannabis use at baseline and psychotic symptoms at follow up. Figures are odds ratios (95\% confidence intervals)

\begin{tabular}{|c|c|c|c|c|c|}
\hline \multirow{2}{*}{$\begin{array}{l}\text { Cannabis exposure at } \\
\text { baseline }\end{array}$} & \multicolumn{4}{|c|}{ Any psychotic symptom } & \multirow{2}{*}{$\begin{array}{c}\text { At least two psychotic } \\
\text { symptoms } \\
\text { Adjusted }^{\star}\end{array}$} \\
\hline & Unadjusted & Adjusted* & Additional adjustment† & Additional adjustment‡ & \\
\hline Any use ( $\geq 5$ times) & 1.79 (1.36 to 2.36$)$ & 1.69 (1.26 to 2.25$)$ & 1.67 (1.13 to 2.46 ) & 1.53 (1.13 to 2.07$)$ & 2.23 (1.52 to 3.29$)$ \\
\hline
\end{tabular}

${ }^{*}$ Age, sex, socioeconomic status, urbanicity, childhood trauma, and predisposition for psychosis at baseline.

†Also adjusted for other drug use, tobacco, and alcohol.

łAlso adjusted for predisposition for psychosis at follow up and depression at baseline and follow up. 
Table 3 Associations between frequency of cannabis use at baseline and any psychotic symptoms. Figures are odds ratios (95\% confidence intervals)

\begin{tabular}{lcc}
$\begin{array}{l}\text { Cumulative frequency of } \\
\text { cannabis use }\end{array}$ & Unadjusted & Adjusted $^{*}$ \\
\hline None $\dagger$ & 1 & 1 \\
\hline$<1 /$ month & $1.01(0.55$ to 1.86$)$ & $0.99(0.53$ to 1.84$)$ \\
\hline $3-4$ times/month & $1.56(0.91$ to 2.68$)$ & 1.50 (0.86 to 2.62$)$ \\
\hline $1-2$ times/week & $2.28(1.28$ to 4.09$)$ & $1.95(1.07$ to 3.55$)$ \\
\hline $3-4$ times/week & $3.07(1.49$ to 6.31$)$ & 2.44 (1.16 to 5.13$)$ \\
\hline Almost daily & $2.57(1.52$ to 4.34$)$ & 2.23 (1.30 to 3.84$)$ \\
\hline Linear trendł & $1.24(1.15$ to 1.35$)$ & 1.20 (1.10 to 1.31$)$ \\
\hline
\end{tabular}

*Adjusted for age, sex, socioeconomic, urbanicity, childhood trauma, and predisposition for psychosis at baseline.

†Reference category.

flncrease in risk with one unit change in cannabis frequency.

Pfister, Victoria Reed, Andrea Schreier, and Petra Zimmermann. Scientific advisers are Jules Angst (Zurich), Jürgen Margraf (Basel), Günther Esser (Potsdam), Kathleen Merikangas (NIMH, Bethesda), Jim van Os (Maastricht), and Ron Kessler (Harvard, Boston).

Contributors: H-UW and RL were the principal investigators of the study. $\mathrm{CH}$ analysed the data in collaboration with LK, JvO, and JS. CH drafted the paper. All authors contributed to subsequent drafts and the final version. $\mathrm{JvO}$ is guarantor for the paper. CK contributed to drafts of the paper and the final version.

Funding: The EDSP study is funded by grants of the German Ministry of Research, Education and Technology 01EB9405/6 and 01EB9901/6 and the Deutsche Forschungsgemeinschaft (DFG). This paper is also part of NIH-grant "Longitudinal analysis of psychiatric risk factors for SUD" (RO1DA016977-01, PI: E. Costello, HUWittchen Co-PI).

Competing interests: None declared.

Ethical approval: The local ethics committee approved the study.

1 Andreasson S, Allebeck P, Engstrom A, Rydberg U. Cannabis and schizophrenia. A longitudinal study of Swedish conscripts. Lancet 1987;ii:1483-6.

2 van Os J, Bak M, Hanssen M, Bijl RV, de Graaf R, Verdoux H. Cannabis use and psychosis: a longitudinal population-based study. Am J Epidemiol 2002;156:319-27.

3 Degenhardt L, Hall W. The association between psychosis and problematical drug use among Australian adults: findings from the national survey of mental health and wellbeing. Psychol Med 2001;31:659-68.

4 Macleod J, Oakes R, Copello A, Crome I, Egger M, Hickman M, et al. Psychological and social sequelae of cannabis and other illicit drug use by young people: a systematic review of longitudinal, general population studies. Lancet 2004;363:1579-88.

5 Khantzian EJ. The self-medication hypothesis of addictive disorders: focus on heroin and cocaine dependence. Am J Psychiatry 1985;142:1259-64.

6 Verdoux H, Gindre C, Sorbara F, Tournier M, Swendsen JD. Effects of cannabis and psychosis vulnerability in daily life: an experience sampling test study. Psychol Med 2003:33:23-32

7 Lieb R, Isensee B, von Sydow K, Wittchen HU. The early developmental stages of psychopathology study (EDSP): a methodological update. Eur Addict Res 2000;6:170-82

8 Poulton R, Caspi A, Moffitt TE, Cannon M, Murray R, Harrington H. Children's self-reported psychotic symptoms and adult schizophreniform disorder: a 15-year longitudinal study. Arch Gen Psychiatry 2000;57:1053-8

9 Breslow NE, Day NE. Statistical methods in cancer research. Vol 2. The design and analysis of cohort studies. New York: Oxford University Press, 1994.

10 Wittchen HU, Lachner G, Wunderlich U, Pfister H. Test-retest reliability of the computerized DSM-IV version of the Munich-Composite International Diagnostic Interview (M-CIDI). Soc Psychiatry Psychiatr Epidemiol 1998;33:568-78.

11 Derogatis JR. SCL-90-R: administration, scoring, and procedures manual-II. Towson: Clinical Psychometric Research, 1983.

12 Sharpley MS, Hutchinson G, Murray RM, McKenzie K. Understanding the excess of psychosis among the African-Caribbean population in England: review of current hypotheses. Br J Psychiatry 2001;178(suppl 40):S60-8.

\section{What is already known on this topic}

It is generally accepted that cannabis use is strongly associated with psychosis

We do not know whether the association is causal or whether those with a predisposition for psychosis are particularly at risk

\section{What this study adds}

Cannabis use in young people moderately increased the risk of developing psychotic symptoms

The risk for the onset of symptoms was much higher in young people with a predisposition for psychosis

Predisposition psychosis at baseline did not predict cannabis use at follow up, thus refuting the self medication hypothesis

13 Read J, Ross CA. Psychological trauma and psychosis: another reason why people diagnosed schizophrenic must be offered psychological therapies. JAm Acad Psychoanal Dyn Psychiatry 2003:31:247-68.

14 Darroch J. Biologic synergism and parallelism. Am J Epidemiol 1997;145:661-8.

15 Van Os J, Hanssen M, Bak M, Bijl RV, Vollebergh W. Do urbanicity and familial liability coparticipate in causing psychosis? Am J Psychiatry 2003;160:477-82.

16 Johns LC, van Os J. The continuity of psychotic experiences in the general population. Clin Psychol Rev 2001;21:1125-41.

17 Kendler KS, Thacker L, Walsh D. Self-report measures of schizotypy as indices of familial vulnerability to schizophrenia. Schizophr Bull 1996;22:511-20.

18 Verdoux H, Sorbara F, Gindre C, Swendsen JD, van Os J. Cannabis use and dimensions of psychosis in a nonclinical population of female subjects. Schizophr Res 2003;59:77-84.

19 Chen J, Paredes W, Lowinson JH, Gardner EL. Delta 9-tetrahydrocannabinol enhance presynaptic dopamine efflux in medial prefrontal cortex. Eur J Pharmacol 1990;190:259-62.

20 Dean B, Sundram S, Bradbury R, Scarr E, Copolov D. Studies on [3H]CP-55940 binding in the human central nervous system: regional specific changes in density of cannabinoid-1 receptors associated with schizophrenia and cannabis use. Neuroscience 2001;103:9-15.

21 Leweke FM, Giuffrida A, Wurster U, Emrich HM, Piomelli D. Elevated endogenous cannabinoids in schizophrenia. Neuroreport 1999;10:1665-9.

22 Schneider M, Koch M. Chronic pubertal, but not adult chronic cannabinoid treatment impairs sensorimotor gating, recognition memory, and the performance in a progressive ratio task in adult rats. Neuropsychopharmacology 2003;28:1760-9.

(Accepted 27 September 2004)

doi $10.1136 /$ bmj.38267.664086.63

Department of Psychiatry and Neuropsychology, South Limburg Mental Health Research and Teaching Network, EURON, Maastricht University, PO BOX 616 6200 MD Maastricht, Netherlands

Cécile Henquet research psychologist

Lydia Krabbendam lecturer

Janneke Spauwen research psychologist

Charles Kaplan senior lecturer

Jim van Os professor

Max Planck Institute of Psychiatry, Clinical Psychology and Epidemiology Unit, Kraepelinstrasse 2, D-80804 Munich, Germany

Roselind Lieb private lecturer

Institute of Clinical Psychology and Psychotherapy, Technical University of

Table 4 Interactions between any cannabis use and predisposition for psychosis

\begin{tabular}{|c|c|c|c|c|c|}
\hline \multirow[b]{2}{*}{ Cannabis use at baseline } & \multirow{2}{*}{$\begin{array}{l}\text { No with psychosis } \\
\text { outcome }^{*}\end{array}$} & \multirow{2}{*}{$\begin{array}{c}\text { No without psychosis } \\
\text { outcome* }\end{array}$} & \multirow{2}{*}{$\begin{array}{l}\text { Risk of psychotic symptoms at } \\
\text { follow up }\end{array}$} & \multicolumn{2}{|c|}{ Difference in risk } \\
\hline & & & & Unadjusted & Adjusted† (95\% Cl) \\
\hline \multicolumn{6}{|c|}{ No predisposition for psychosis at baseline } \\
\hline None & 294 & 1642 & $15 \%$ & $6 \%$ & $5.6 \% \quad(0.4$ to 10.8$) P=0.033$ \\
\hline Any ( $\geq 5$ times) & 59 & 216 & $21 \%$ & & \\
\hline \multicolumn{6}{|c|}{ Predisposition for psychosis at baselineł } \\
\hline None & 47 & 133 & $26 \%$ & $25 \%$ & $23.8 \%$ (7.9 to 39.7) $\mathrm{P}=0.003$ \\
\hline Any ( $\geq 5$ times) & 23 & 22 & $51 \%$ & & \\
\hline
\end{tabular}

*Numbers total 2436 because of one missing value on predisposition for psychosis at baseline.

†Age, sex, socioeconomic status, urbanicity, childhood trauma, and predisposition for psychosis at follow up. Test for additive interaction $18.2 \%$ adjusted difference in risk (95\% confidence interval 1.6 to 34.8 ), $P=0.032$ (tests whether risk difference in "predisposition" group is significantly greater than risk difference in "no predisposition" group).

†Total score $\geq 90$ th centile on "paranoid ideation" and "psychoticism" subscales of symptom checklist. 
